\title{
TOPOLOGIES DEFINED BY SOME INVARIANT PSEUDODISTANCES
}

\author{
THEODORE J. BARTH \\ Department of Mathematics, University of California \\ Riverside, California 92521, U.S.A. \\ E-mail: tjb@math.ucr.edu
}

Complex analysis deals with holomorphic mappings between complex spaces. We ought to allow complex spaces to be infinite-dimensional and to have singularities. There are well-developed theories in the finite-dimensional case (see e.g. [GR], [Lo]) and for domains in certain kinds of topological vector spaces (see e.g. [Di], [FV], [He]). The most obvious approach to infinite-dimensional complex spaces is too general to yield sensible results [Do], but there has been progress $[\mathrm{Au}]$ in the study of (possibly infinite-dimensional, possibly singular) semi-Fredholm-analytic spaces. In any case, I shall assume that a complex space carries the structure of a Hausdorff space, which I shall call its underlying topology; in the finite-dimensional case I shall assume that the underlying topology is paracompact. This note discusses some foundational questions and summarizes what we know about the relationships between the underlying topology and the topologies induced by the classical pseudodistances of Carathéodory and Kobayashi.

Let $\operatorname{Hol}(X, Y)$ denote the set of all holomorphic mappings from the complex space $X$ into the complex space $Y$; I shall assume that these mappings are continuous with respect to the underlying topologies of $X$ and $Y$. Let $\mathbb{D}$ denote the open unit disc in $\mathbb{C}$. A mapping $f \in \operatorname{Hol}(\mathbb{D}, X)$ is called an analytic disc in $X$. I shall also assume that a complex space has the property that any two points can be connected by (the images of) a finite chain of analytic discs in the space; in the finite-dimensional case this is equivalent to connectedness in the topological sense [K70, pp. 97-98], [La, pp. 15-16].

1991 Mathematics Subject Classification: Primary 32H15; Secondary 51K05.

The paper is in final form and no version of it will be published elsewhere. 
1. The Carathéodory pseudodistance. The Carathéodory pseudodistance [C, pp. 78-79] between the points $p$ and $q$ of the complex space $X$ is

$$
c_{X}(p, q)=\sup \left\{\tanh ^{-1}(f(q)): f \in \operatorname{Hol}(X, \mathbb{D}), f(p)=0, f(q) \geq 0\right\} .
$$

Obviously $0 \leq c_{X}(p, q) \leq \infty$. Moreover $c_{\mathbb{D}}=\rho$, the Poincaré distance. When a Vitali-Montel Theorem holds for $X$, one can use a "normal families" argument to show that the supremum is attained, hence that $c_{X}(p, q)$ is finite. In the finitedimensional singular case, a suitable Vitali Theorem was proved independently by Gunning [G] and Andreotti and Stoll [AnS, Proposition 4, pp. 326-328]; a Montel Theorem for domains in locally convex spaces was given in [DiTV, Lemme 1.2, pp. 516-517]. Alternatively, for any analytic disc $f: \mathbb{D} \rightarrow X$ it follows from the the Schwarz Lemma that $\rho(0, a) \geq c_{X}(f(0), f(a))$; connecting $p$ and $q$ by a chain of analytic discs shows that $c_{X}(p, q)$ is finite.

The first topological chore is to check whether $c_{X}: X \times X \rightarrow[0, \infty)$ is continuous (with respect to the underlying topology). In view of the triangle inequality, this amounts to the continuity of $c_{X}(p, \cdot)$ at each $p \in X$. The argument given by Carathéodory [C, Satz 6, pp. 85-86] for bounded domains in $\mathbb{C}^{2}$ works in great generality as long as $X$ is nonsingular at $p$ : take a balanced convex domain $B$ with center $p$ with respect to a chart of $X$; the inequality $c_{B}(p, q) \geq c_{X}(p, q)$ and an explicit computation of $c_{B}(p, q)$ show that $c_{X}(p, q) \rightarrow 0$ as $q \rightarrow p$. Alternatively, when a Vitali-Montel Theorem holds, one can give a normal families argument. Recently V. Aurich [Au, Lemma 5.4, p. 240] has shown that $c_{X}$ is continuous when $X$ is a (possibly infinite-dimensional, possibly singular) semi-Fredholm-analytic space.

In an abstract sense the following completely settles all questions about the topology induced by $c_{X}$.

Proposition 1.1. Let $X$ be a complex space. Let $\mathrm{H}^{\infty}(X)$ denote the space of bounded (complex-valued) holomorphic functions on $X$ equipped with the supremum norm $\|\cdot\|$, and let $\mathrm{H}^{\infty}(X)^{*}$ denote the space of bounded linear functionals on $\mathrm{H}^{\infty}(X)$ equipped with the dual norm $\|\cdot\|^{*}$. Let $\tau: X \rightarrow \mathrm{H}^{\infty}(X)^{*}$ be the evaluation map defined by $\tau(p)(f)=f(p)$ for $p \in X, f \in \mathrm{H}^{\infty}(X)$. Then

(a) $c_{X}(p, q)=2 \tanh ^{-1}\left(\frac{1}{2}\|\tau(p)-\tau(q)\|^{*}\right)$ for all $p, q \in X$;

(b) $c_{X}$ is a distance if and only if $\tau$ is one-to-one;

(c) $c_{X}$ is continuous if and only if $\tau$ is continuous;

(d) the topology induced by $c_{X}$ is $\left\{\tau^{-1}(U): U\right.$ is open in $\left.\mathrm{H}^{\infty}(X)^{*}\right\}$;

(e) $c_{X}$ induces the underlying topology if and only if $\tau$ is an embedding.

Obviously (b) through (e) follow from (a), though it is easy to check them independently of it. The formula in (a) was stated in [EaH, Lemma 4, p. 65]; its proof relies on a neat argument given by J. Lewittes [Le, p. 1090]:

$$
\begin{aligned}
\|\tau(p)-\tau(q)\|^{*} & =\sup \left\{|f(p)-f(q)|: f \in \mathrm{H}^{\infty}(X),\|f\| \leq 1\right\} \\
& =\sup \{|f(p)-f(q)|: f \in \operatorname{Hol}(X, \mathbb{D})\} \\
& =\sup \{|f(p)-f(q)|: f \in \operatorname{Hol}(X, \mathbb{D}), f(q)=-f(p) \geq 0\}
\end{aligned}
$$




$$
\begin{aligned}
& =2 \sup \{f(q): f \in \operatorname{Hol}(X, \mathbb{D}), f(q)=-f(p) \geq 0\} \\
& =2 \tanh (\sup \{\rho(0, f(q)): f \in \operatorname{Hol}(X, \mathbb{D}), f(q)=-f(p) \geq 0\}) \\
& =2 \tanh \left(\frac{1}{2} \sup \{\rho(f(p), f(q)): f \in \operatorname{Hol}(X, \mathbb{D}), f(q)=-f(p) \geq 0\}\right) \\
& =2 \tanh \left(\frac{1}{2} c_{X}(p, q)\right) .
\end{aligned}
$$

The key point here is the third equality, which says that $|f(p)-f(q)|$ will be maximized by choosing $f(p)$ and $f(q)$ symmetrically about 0 .

In connection with Proposition 1.1(e) there are some concrete positive results.

Proposition 1.2. Each of the following is a sufficient condition for $c_{X}$ to induce the underlying topology:

(a) $X$ is a bounded domain in a Banach space;

(b) $X$ is a domain in $\mathbb{C}$ and $c_{X}$ is a distance;

(c) $X$ is a relatively compact domain in a Stein space;

(d) $X$ is finite-dimensional, $c_{X}$ is a distance, and the closed Carathéodory ball $\left\{q \in X: c_{X}(p, q) \leq r\right\}$ is compact (with respect to the underlying topology) for every $p \in X$ and every $r>0$.

The proof of (a) is easy: if $U$ is a neighborhood of a point $p$ in $X$, we take a ball $B$ centered at $p$ and containing $X$, compute $c_{B}(p, q)$ to find a radius $\delta$ for which $\left\{q \in X: c_{B}(p, q)<\delta\right\} \subset U$, and note that $c_{X}(p, q) \geq c_{B}(p, q)$. A slick elementary proof of (b) can be found in [JP91, Proposition 1.3, p. 170]. Proofs of (c) and (d) were given by N. Sibony [Sib, pp. 222-223].

Recently Mikihiro Hayashi [Ha] constructed a Riemann surface (a one-dimensional complex manifold) $X$ for which the mapping $\tau$ of Proposition 1.1 is one-toone but not an embedding. In view of parts (b) and (e) of Proposition 1.1, JeanPierre Vigué observed that this Riemann surface provides an example of a complex space for which the Carathéodory pseudodistance is a distance but does not induce the underlying topology. Marek Jarnicki, Peter Pflug, and Vigué [JPV91] applied some general theorems to establish the existence of a domain in $\mathbb{C}^{3}$ with the same property. Indeed, a Remmert-Narasimhan-Bishop embedding realizes Hayashi's example as closed complex submanifold of $\mathbb{C}^{3}$; using Siu's theorem [Siu], it is easy to construct such a domain containing this submanifold. The experts [ElG, p. 128] seem to believe that every open Riemann surface will embed in $\mathbb{C}^{2}$; once this has been proved there will be a corresponding example of a domain in $\mathbb{C}^{2}$.

Even when a Carathéodory distance induces the underlying topology, recent examples of Riemann surfaces and bounded pseudoconvex domains [JPV92] show that the closure of an open Carathéodory ball may be a proper subset of the corresponding closed Carathéodory ball.

2. Inner pseudodistances. Kobayashi [K73, p. 484] suggested the idea of applying the metric space theory of inner distances, as codified by Rinow [Ri], to the Carathéodory distance. This theory, which goes back at least to the work of 
Menger [M, p. 492], measures distance by the infimum of the lengths of (continuous) curves joining two given points. In view of the Hayashi-Jarnicki-Pflug-Vigué examples, this construction may use curves that do not conform to our intuition of a "curve" in a complex space.

In hopes of clarifying the nature of the difficulties, I shall now introduce some terminology. A path in the set $X$ is a mapping $\gamma:[a, b] \rightarrow X$, where $a$ and $b$ are real numbers with $a \leq b$; the values $\gamma(a)$ and $\gamma(b)$ are called the endpoints of $\gamma$. Let me emphasize that $X$ is only a set and there is no continuity assumption. A network on $X$ is a collection $\mathfrak{N}$ of paths in $X$ satisfying:

- whenever $p$ and $q$ are points of $X$ there is a path in $\mathfrak{N}$ with endpoints $p, q$;

- if $\gamma:[a, b] \rightarrow X$ and $a \leq t \leq b$, then $\gamma \in \mathfrak{N}$ if and only if $\left.\gamma\right|_{[a, t]} \in \mathfrak{N}$ and $\left.\gamma\right|_{[t, b]} \in \mathfrak{N}$

- if $\gamma \in \mathfrak{N}$ and $A: \mathbb{R} \rightarrow \mathbb{R}$ is a nonconstant affine function, then $\gamma \circ A \in \mathfrak{N}$.

Intuitively, a network is closed under path product, reversal, and restriction. Finally, a gigadistance on the set $X$ is a function $D: X \times X \rightarrow[0, \infty]$ satisfying the usual axioms for a pseudodistance:

(1) $D(p, p)=0$ for all $p \in X$,

(2) $D(p, q)=D(q, p)$ for all $p, q \in X$,

(3) $D(p, q)+D(q, r) \geq D(p, r)$ for all $p, q, r \in X$.

Once we accept these definitions, it is reasonable to define the length of the path $\gamma:[a, b] \rightarrow X$ relative to the gigadistance $D$ to be

$$
L(\gamma, D)=\sup \left\{\sum_{j=1}^{k} D\left(\gamma\left(t_{j-1}\right), \gamma\left(t_{j}\right)\right): k \geq 1, a=t_{0} \leq t_{1} \leq \cdots \leq t_{k}=b\right\} .
$$

Of course $0 \leq L(\gamma, D) \leq \infty$. Setting

$$
D^{\mathfrak{N}}(p, q)=\inf \{L(\gamma, D): \gamma \in \mathfrak{N} \text { and } \gamma \text { has endpoints } p, q\}
$$

gives another gigadistance $D^{\mathfrak{N}} \geq D$; it may happen that $D^{\mathfrak{N}}$ is infinite even when $D$ is a (finite) distance and the paths in $\mathfrak{N}$ are continuous with respect to $D$. We say that the gigadistance $D$ is inner for $\mathfrak{N}$ if $D^{\mathfrak{N}}=D$. If $D$ is an arbitrary gigadistance, it is easy to see that $L\left(\gamma, D^{\mathfrak{N}}\right)=L(\gamma, D)$ for every $\gamma \in \mathfrak{N}$, so $D^{\mathfrak{N}}$ is inner for $\mathfrak{N}$. The book [Ri] considers the case where $(X, D)$ is a pathwise connected metric space and $\mathfrak{N}$ consists of all paths that are continuous with respect to $D$. To conclude the general discussion, here is a simple topological fact.

LEMma 2.1. Let $D$ be a continuous distance on the locally compact Hausdorff space $X$. Let $\mathfrak{N}$ be a network on $X$ consisting of mappings that are continuous with respect to the given topology on $X$. If $D$ is inner for $\mathfrak{N}$, then $D$ induces the given topology.

Now let $X$ be a complex space such that $c_{X}$ is a continuous pseudodistance (a condition essentially always satisfied - see $\S 1$ ). There are at least four natural networks on $X$ : 
- the network $\mathfrak{C}$ of all paths continuous with respect to $c_{X}$,

- the network $\mathfrak{U}$ of all paths continuous with respect to the underlying topology,

- the network $\mathfrak{V}$ of admissible curves of Venturini [Ve, p. 387],

- the network $\mathfrak{D}$ of all piecewise continuously differentiable paths.

All have been used to construct "inner" Carathéodory (pseudo)distances:

- $c_{X}{ }^{\mathfrak{C}}$, denoted by $c_{X}^{\prime}$ in [K73, p. 484];

- $c_{X}{ }^{\mathfrak{U}}$, denoted by $c_{X}^{i}$ in [K76, p. 364], [B77, p. 49], [PS, p. 67];

- $c_{X} \mathfrak{V}$, denoted by $c_{X}^{i}$ in [Ve, p. 390];

- $c_{X} \mathfrak{D}$, denoted by $\widetilde{C}_{X}$ in [Di, p. 53].

(I have guessed some authors' intent.) Clearly $\mathfrak{C} \supset \mathfrak{U} \supset \mathfrak{V} \supset \mathfrak{D}$, so $c_{X} \leq c_{X}{ }^{\mathfrak{C}} \leq$ $c_{X} \mathfrak{U} \leq c_{X} \mathfrak{V} \leq c_{X}^{\mathfrak{D}}$. It is quite usual to have $c_{X} \neq c_{X}{ }^{\mathfrak{C}}$ (see e.g. [B77], [Vi83], [JP90]). Jean-Pierre Vigué has noted that the 2-dimensional complex space constructed in [Vi84] satisfies $c_{X} \mathfrak{C} \neq c_{X} \mathfrak{U}$. If I interpret the author(s) correctly, [K76, Theorem 2.6(2), p. 364] (resp. [PS, p. 67]) states that $c_{X}{ }^{\mathfrak{U}}=c_{X}{ }^{\mathfrak{D}}$ whenever $X$ is a finite-dimensional complex space (resp. manifold); Jarnicki and Pflug [JP91, p. 170] note that this is true when $X$ is a bounded domain in $\mathbb{C}^{n}$, but they point out the lack of a proof in the general case. While it may be counterintuitive for a ragged curve to be short, it is conceivable that a trip along such a curve may encounter little resistance from $c_{X}$.

The arguments in $\S 1$ show that $c_{X} \mathfrak{D}(p, q)$ is finite and also that $c_{X} \mathfrak{D}(p, \cdot)$ is continuous at each nonsingular point $p$. In the finite-dimensional singular case continuity follows from comparison with the Kobayashi distance (see $\S 3$ ) or by noting that $c_{X} \mathfrak{D}$ coincides with the integrated form of the Carathéodory-Reiffen metric [Re, Satz 2, p. 321].

THEOREM 2.2. Each of the following is a sufficient condition for $c_{X} \mathfrak{U}$ (resp. $\left.c_{X} \mathfrak{D}\right)$ to induce the underlying topology:

(a) $X$ is a bounded domain in a Banach space;

(b) $X$ is finite-dimensional and $c_{X} \mathfrak{U}\left(r e s p . c_{X} \mathfrak{D}\right)$ is a distance.

The proof of (a) is the same as for Proposition 1.2(a). Of course (b) follows from Lemma 2.1; the only difficult part is continuity at the singular points.

In view of Theorem 2.2, it is natural to look at unbounded domains in the Hilbert space $\ell^{2}$ of sequences of complex numbers $x=\left(x_{\nu}\right)_{\nu=1}^{\infty}$ satisfying $\|x\|=$ $\sqrt{\sum\left|x_{\nu}\right|^{2}}<\infty$. Following [FV, pp. 93-94], set

$$
X=\left\{x \in \ell^{2}: \sup _{\nu}\left|x_{\nu}\right| / \nu<1\right\} .
$$

Then $c_{X} \mathfrak{U}^{\mathfrak{U}}$ and $c_{X} \mathfrak{D}$ are distances, $x^{(n)}=\left(\delta_{\nu}^{n+1}\right)_{\nu=1}^{\infty} \in X$, and

$$
c_{X} \mathfrak{U}\left(0, x^{(n)}\right)=c_{X} \mathfrak{D}\left(0, x^{(n)}\right)=\tanh ^{-1}\left(\frac{1}{n+1}\right) \rightarrow 0 \quad \text { as } \quad n \rightarrow \infty .
$$

But $\left\|x^{(n)}\right\|=1$, so these distances cannot induce the underlying (norm) topology. 
3. The Kobayashi pseudodistance. The definition of the Kobayashi pseudodistance [K67, p. 462], as explained by Royden [Ro], involves dualizing the Carathéodory construction to obtain an object that may not be a pseudodistance, and then applying a general technique.

At the risk of overdoing the jargon, let us say that a paradistance on the set $X$ is a function $D: X \times X \rightarrow[0, \infty]$ satisfying:

(1) $D(p, p)=0$ for all $p \in X$,

(2) $D(p, q)=D(q, p)$ for all $p, q \in X$.

Lemma 3.1. Let $D$ be a paradistance on the set $X$. For $p, q \in X, k=1,2,3, \ldots$, set

Then

$$
D^{(k)}(p, q)=\inf \left\{\sum_{j=1}^{k} D\left(p_{j-1}, p_{j}\right): p_{0}=p, p_{k}=q\right\} .
$$

(a) $D^{(k)}$ is a paradistance on $X$;

(b) $D=D^{(1)} \geq D^{(2)} \geq D^{(3)} \geq \ldots$;

(c) $D^{(\infty)}=\inf _{k} D^{(k)}=\lim _{k \rightarrow \infty} D^{(k)}$ is a gigadistance on $X$.

In other words, $D^{(\infty)}$ is the largest gigadistance on $X$ bounded above by $D$.

Now let $X$ be a complex space. The one-disc Kobayashi paradistance between the points $p$ and $q$ of $X$ is

$$
\delta_{X}(p, q)=\inf \left\{\tanh ^{-1}(a): 0 \leq a<1, f \in \operatorname{Hol}(\mathbb{D}, X), f(0)=p, f(a)=q\right\} .
$$

It may happen that the set in this formula is empty, in which case the infimum is defined to be $\infty$. But if $X$ is an $n$-dimensional complex manifold, a theorem of Fornaess and Stout [FS77] (resp. [FS82]) asserts the existence of a holomorphic mapping of $\mathbb{D}^{n}$ (resp. the $n$-dimensional ball) onto $X$, whence $\delta_{X}$ is finite. This is also true if $X$ is a domain in a Banach space [Di, pp. 49-50]. The infimum is not usually attained, but it will be if $X$ is taut [W, Definition 1.2, p. 199], [DiT, Definition 6.2, p. 359]. The Kobayashi pseudodistance on $X$ is

$$
d_{X}=\delta_{X}^{(\infty)}
$$

If $f \in \operatorname{Hol}(\mathbb{D}, X)$ and $0 \leq a<1$ we have

$$
d_{X}(f(0), f(a)) \leq \delta_{X}(f(0), f(a)) \leq \tanh ^{-1}(a)=\rho(0, a) .
$$

Using a chain of discs one sees that $d_{X}$ is finite. Moreover the geodesic properties of $\rho$ give $L\left(\left.f\right|_{[0, a]}, d_{X}\right) \leq \rho(0, a)$. It follows that $d_{X}{ }^{\mathfrak{A}} \leq \delta_{X}$, where $\mathfrak{A}$ is the smallest network on $X$ containing $\left\{\left.f\right|_{[0, a]}: 0 \leq a<1, f \in \operatorname{Hol}(\mathbb{D}, X)\right\}$. Thus $d_{X}=d_{X}^{\mathfrak{U}}=d_{X}^{\mathfrak{V}}=d_{X}^{\mathfrak{D}}=d_{X}^{\mathfrak{A}}$.

The argument in $\S 1$ shows that $d_{X}(p, \cdot)$ is continuous at each nonsingular point $p$. The only simple proof I know for continuity in the finite-dimensional singular case uses a Hironaka resolution of singularities [Hi], [HiR]; see [B72], [La, Proposition 1.7, pp. 17-18]. Possibly the new metric of Kobayashi [K90] could be helpful in this regard. 
Let $\mathfrak{K}$ be the network of all paths continuous with respect to $d_{X}$. When $d_{X}$ is continuous, we have $\mathfrak{K} \supset \mathfrak{U}$, so $d_{X}=d_{X} \mathfrak{K}$, i.e. $d_{X}$ is inner for $\mathfrak{K}[K 73$, pp. 483-484].

THEOREM 3.2. Each of the following is a sufficient condition for $d_{X}$ to induce the underlying topology:

(a) $X$ is a bounded domain in a Banach space;

(b) $X$ is finite-dimensional and $d_{X}$ is a distance.

The proof is identical to that of Theorem 2.2. Moreover, for the unbounded domain $X$ in $\ell^{2}$ constructed at the end of $\S 2, d_{X}$ is a distance that does not induce the underlying topology [FV, pp. 93-94].

\section{References}

[AnS] A. Andreotti and W. Stoll, Extension of holomorphic maps, Ann. of Math. (2) 72 (1960), 312-349.

[Au] V. Aurich, Bounded analytic sets in Banach spaces, Ann. Inst. Fourier (Grenoble) 36 (1986), 229-243.

[B72] T. J. Barth, The Kobayashi distance induces the standard topology, Proc. Amer Math. Soc. 35 (1972), 439-441.

[B77] —, Some counterexamples concerning intrinsic distances, ibid. 66 (1977), 49-53.

[C] C. Carathéodory, Über das Schwarzsche Lemma bei analytischen Funktionen von zwei komplexen Veränderlichen, Math. Ann. 97 (1926), 76-98.

[Di] S. Dineen, The Schwarz Lemma, Oxford University Press, 1989.

[DiT] S. Dineen and R. M. Timoney, Complex geodesics on convex domains, in: Progress in Functional Analysis, North-Holland, 1992, 333-365.

[DiTV] S. Dineen, R. M. Timoney et J.-P. Vigué, Pseudodistances invariantes sur les domaines d'un espace localement convexe, Ann. Scuola Norm. Sup. Pisa Cl. Sci. (4) 12 (1985), 515-529.

[Do] A. Douady, A remark on Banach analytic spaces, in: Symposium on Infinite Dimensional Topology, Princeton University Press, 1972, 41-42.

[EaH] C. J. Earle and R. S. Hamilton, A fixed point theorem for holomorphic mappings, in: Global Analysis (Berkeley, Calif. 1968), Proc. Sympos. Pure Math. 16, Amer. Math. Soc., 1970, 61-65.

[ElG] Y. Eliashberg and M. Gromov, Embeddings of Stein manifolds of dimension $n$ into the affine space of dimension 3n/2 +1, Ann. of Math. (2) 136 (1992), 123-135.

[FS77] J. E. Fornaess and E. L. Stout, Spreading polydiscs on complex manifolds, Amer J. Math. 99 (1977), 933-960.

[FS82] - - - Regular holomorphic images of balls, Ann. Inst. Fourier (Grenoble) 32 (1982), 23-36.

[FV] T. Franzoni and E. Vesentini, Holomorphic Maps and Invariant Distances, North-Holland, 1980.

[G] R. C. Gunning, On Vitali's theorem for complex spaces with singularities, J. Math Mech. 8 (1959), 133-141.

[GR] R. C. Gunning and H. Rossi, Analytic Functions of Several Complex Variables, Prentice-Hall, 1965.

[Ha] M. Hayashi, The maximal ideal space of the bounded analytic functions on a Riemann surface, J. Math. Soc. Japan 39 (1987), 337-344.

[He] M. Hervé, Analyticity in Infinite Dimensional Spaces, Walter de Gruyter, 1989. 
[Hi] H. Hironaka, Resolution of singularities of an algebraic variety over a field of characteristic zero, Ann. of Math. (2) 79 (1964), 109-326.

[HiR] H. Hironaka and H. Rossi, On the equivalence of imbeddings of exceptional complex spaces, Math. Ann. 156 (1964), 313-333.

[JP90] M. Jarnicki and P. Pflug, The simplest example for the non-innerness of the Carathéodory distance, Results Math. 18 (1990), 57-59.

[JP91] —, -, Invariant pseudodistances and pseudometrics-completeness and the product property, Ann. Polon. Math. 55 (1991), 169-189.

[JPV91] M. Jarnicki, P. Pflug and J.-P. Vigué, The Carathéodory distance does not define the topology - the case of domains, C. R. Acad. Sci. Paris Sér. I Math. 312 (1991), 77-79.

[JPV92] -, -, A remark on Carathéodory balls, Arch. Math. (Basel) 58 (1992), 595-598.

[K67] S. Kobayashi, Invariant distances on complex manifolds and holomorphic mappings, J. Math. Soc. Japan 19 (1967), 460-480.

[K70] - Hyperbolic Manifolds and Holomorphic Mappings, Marcel Dekker, 1970.

[K73] - Some remarks and questions concerning the intrinsic distance, Tôhoku Math. J. (2) 25 (1973), 481-486.

[K76] - Intrinsic distances, measures and geometric function theory, Bull. Amer. Math. Soc. 82 (1976), 357-416.

[K90] -, A new invariant infinitesimal metric, Internat. J. Math. 1 (1990), 83-90.

[La] S. Lang, Introduction to Complex Hyperbolic Spaces, Springer, 1987.

[Le] J. Lewittes, A note on parts and hyperbolic geometry, Proc. Amer. Math. Soc. 17 (1966), 1087-1090.

[Lo] S. Łojasiewicz, Introduction to Complex Analytic Geometry, Birkhäuser, 1991.

[M] K. Menger, Untersuchungen über allgemeine Metrik. IV. Zur Metrik der Kurven, Math. Ann. 103 (1930), 466-501.

[PS] E. A. Poletskiı̌ and B. V. Shabat, Invariant metrics, in: Complex AnalysisSeveral Variables 3, VINITI, Moscow, 1986 (in Russian); English transl.: Several Complex Variables III, Encyclopaedia Math. Sci. 9, Springer, 1989, 63-111.

[Re] H.-J. Reiffen, Die Carathéodorysche Distanz und ihre zugehörige Differentialmetrik, Math. Ann. 161 (1965), 315-324.

[Ri] W. Rinow, Die innere Geometrie der metrischen Räume, Springer, 1961.

[Ro] H. L. Royden, Remarks on the Kobayashi metric, in: Several Complex Variables II Maryland 1970, Lecture Notes in Math. 185, Springer, 1971, 125-137.

[Sib] N. Sibony, Prolongement des fonctions holomorphes bornées et métrique de Carathéodory, Invent. Math. 29 (1975), 205-230.

[Siu] Y. T. Siu, Every Stein subvariety admits a Stein neighborhood, ibid. 38 (1976), $89-100$.

[Ve] S. Venturini, Pseudodistances and pseudometrics on real and complex manifolds, Ann. Mat. Pura Appl. (4) 154 (1989), 385-402.

[Vi83] J.-P. Vigué, La distance de Carathéodory n'est pas intérieure, Results Math. 6 (1983), 100-104.

[Vi84] - The Carathéodory distance does not define the topology, Proc. Amer. Math. Soc. 91 (1984), 223-224.

[W] H. W u, Normal families of holomorphic mappings, Acta Math. 119 (1967), 193233. 